\title{
Dermoscopic Features of Nevoid Hyperkeratosis of the Nipple and Areola
}

\author{
Conforti Claudio ${ }^{1}$, Dri Arianna ${ }^{1}$, Giuffrida Roberta ${ }^{2}$, Zalaudek Iris $^{1}$, di Meo Nicola ${ }^{1}$
}

1 Dermatology Clinic, University of Trieste, Maggiore Hospital, Piazza dell'Ospitale 1, Trieste, Italy

2 Experimental Dermatology Section, Division of Dermatology, University of Messina, Messina, Italy

Key words: nevoid hyperkeratosis of the nipple and areola, asymptomatic plaques, hyperpigmented plaque, hyperkeratosis

Citation: Conforti C, Dri A, Giuffrida R, Zalaudek I, di Meo N. Dermoscopic features of nevoid hyperkeratosis of the nipple and areola. Dermatol Pract Concept. 2022;12(1):e2022003. DOI: https://doi.org/10.5826/dpc.1201a03

Accepted: April 11, 2021; Published: January 2022

Copyright: $@ 2022$ Conforti et al. This is an open-access article distributed under the terms of the Creative Commons Attribution-NonCommercial License (BY-NC-4.0), https://creativecommons.org/licenses/by-nc/4.0/, which permits unrestricted noncommercial use, distribution, and reproduction in any medium, provided the original authors and source are credited.

Funding: None.

Competing interests: None.

Authorship: All authors have contributed significantly to this publication.

Corresponding author: Claudio Conforti, MD, Dermatology Clinic, University of Trieste, Trieste, Italy.

E-mail: claudioconforti@yahoo.com

\section{Introduction}

Nevoid hyperkeratosis of the nipple and areola (NHNA) is a rare benign condition characterized by verrucous, pigmented, and asymptomatic plaques involving the nipples and areolas. Only a few cases have been reported in literature since this entity was firstly described in 1923 . The etiology is still unknown, but it is hypothesized that it occurs in correlation with hormonal changes because it is more frequent in women during childbearing age, and it worsens during pregnancy.

\section{Case Presentation}

We present the case of a 27-year-old Caucasian woman referred in for consultation to our skin cancer department with a 9-month history of a lesion involving the right nipple and the areola. Examination revealed a hyperpigmented plaque of about $35 \mathrm{~mm}$ in diameter and with well-defined borders (Figure 1B). The lesion was completely asymptom- atic, and the patient denied pain, pruritus or discharge. She was otherwise healthy, and breast examination and general physical examination were negative. Hair, nails, and mucosal sites were normal. The patient was not on medication, and she was not pregnant.

Dermoscopic evaluation $(\times 20$, DermLite $)$ showed the presence of a homogeneous brown network with some hyperkeratotic areas showing yellow-brown scales (Figure 2, $\mathrm{A}$ and $\mathrm{B})$. These dermoscopic findings are in accordance with other previous cases of NHNA, and the diagnosis was later confirmed performing a biopsy.

\section{Discussion and Conclusions}

Cinotti et al reported that at dermoscopic examination the NHNA showed a papillomatous surface with pink homogenous areas, whitish desquamation, red dots, and erosions [1]. Mazzella et al reported a peculiar case of NHNA resembling a pigmented basal cell carcinoma with multiple blue-gray globules and leaf-like areas [2]. 


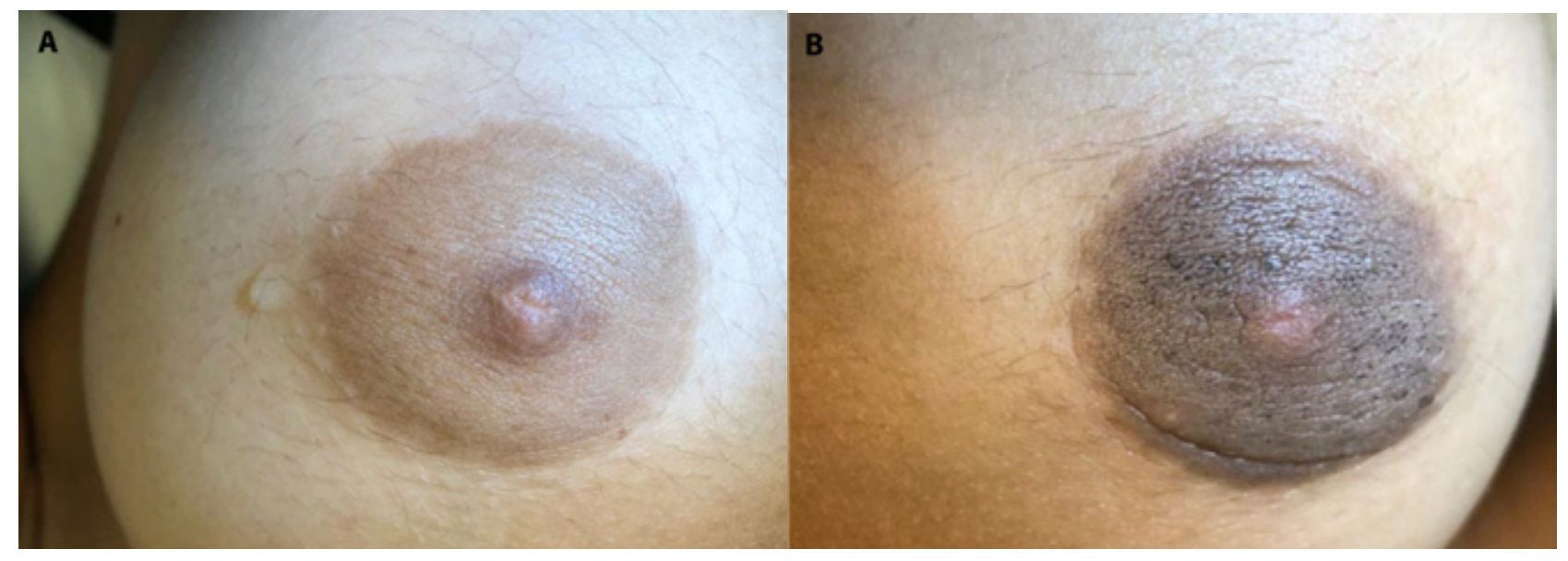

Figure 1. Clinical appearance of the nipples and areolas of a 27-year-old woman. (A) left nipple. (B) right nipple. On the right side a pigmented and asymptomatic plaque involving nipple and areola is showed.
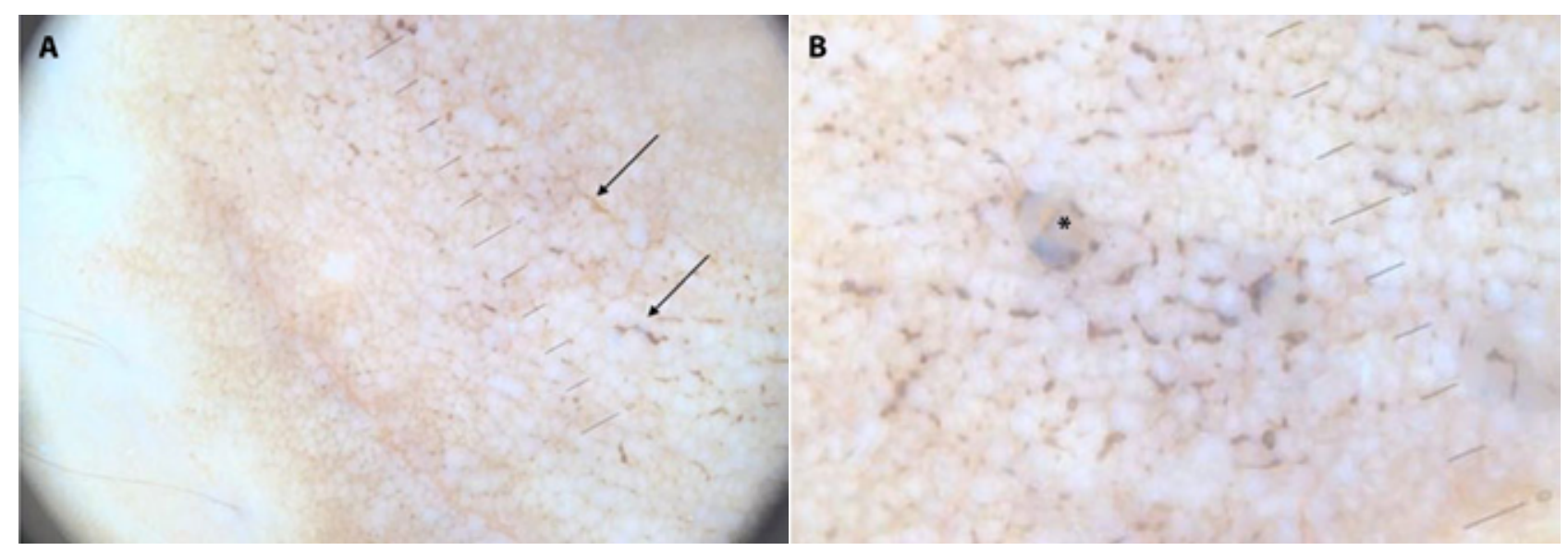

Figure 2. Dermoscopic evaluation (DermLite, 3Gen) (magnification x 20). (A) homogeneous brown network with diffuse hyperkeratotic areas showing yellow-brown scales (black arrows). (B) a blue structureless area (black asterisk).

In the past, skin biopsy was certainly the most accurate way to make a diagnosis of NHNA, but it could create a scar in a delicate and intimate area. Histopathological examination of NHNA can show variable findings such as acanthosis, hyperkeratosis, papillomatosis, keratin plugging, perivascular infiltrates of CD41 lymphocytes, melanophages and plasma cells in the superficial dermis, and hyperpigmentation of the basal layer and dermal fibrosis [1]. Although the histopathological examination remains the gold standard for the diagnosis, sometimes the patient does not accept this option. Dermoscopy offers the possibility of making a correct diagnosis without resorting to incisional biopsy, even if standard dermoscopic diagnostic criteria have not been defined yet. The role of dermoscopy is certainly crucial for the differential diagnosis of areola and nipple lesions, which include seborrheic keratosis, acanthosis nigricans, Darier disease, epidermal nevus, basal cell carcinoma, Bowen disease, Paget disease, contact allergic dermatitis, chronic eczema, dermatophytosis, mycosis fungoides, and reticulate papillomatosis.
All the above pathologies can be diagnosed well with dermoscopy, and for this reason our case underlines the importance of a dermoscopic evaluation of hyperpigmented or nonpigmented lesions of the areola and nipple to reduce excision rate and increase diagnostic accuracy of special-site lesions.

Informed consent: Written informed consent for publication of her clinical details and clinical images was obtained from the patient.

\section{References}

1. Cinotti E, Provvidenziale L, Miracco C, Rubegni P. Treatment of nevoid hyperkeratosis of the areola and nipple with topical tacalcitol: A case report. Dermatol Ther. 2018;31(4):e12602. DOI: 10.1111/dth.12602. PMID: 29624812.

2. Mazzella C, Costa C, Fabbrocini G, et al. Nevoid hyperkeratosis of the nipple mimicking a pigmented basal cell carcinoma. JAAD Case Rep. 2016;2(6):500-501. DOI: 10.1016/j.jdcr.2016.09.007. PMID: 28004028; PMCID: PMC5161776. 